\title{
Treatment of Type 2 (Non-Insulin-Dependent) Diabetic Patients with Diet Alone
}

\author{
J.K. Wales \\ University Department of Medicine, General Infirmary, Leeds, UK
}

\begin{abstract}
Summary. The effects of 3 months' diet therapy on glucose tolerance, plasma insulin response to oral glucose, fasting lipid levels and body weight were measured in 182 Type 2 (non-insulin-dependent) diabetic outpatients with fasting hyperglycaemia before treatment. Overall, there was a $25 \%$ improvement in glucose tolerance, a $58 \%$ increase in plasma insulin response during the glucose tolerance test, a $13.6 \%$ fall in fasting plasma triglyceride levels with no change in fasting cholesterol levels and a $5.1 \%$ fall in body weight. Of the 182 patients, $20 \%$ achieved normal glucose tolerance on diet alone for 3 months. These were mainly men of average age 63 years, who were $20 \%$ above their ideal body weight, and had milder glu-
\end{abstract}

cose intolerance and higher residual insulin response to glucose before treatment than the $16 \%$ of patients who remained poorly controlled. These latter patients were mainly older women, $9 \%$ above their ideal body weight and with more marked glucose intolerance and less insulin response to glucose. These results demonstrate the practical limitations of this type of anti-diabetic therapy, particularly if normal glucose tolerance is a desired therapeutic aim.

Key words: Type 2 diabetes, diet, glucose tolerance, insulin secretion, blood lipids, weight reduction.
With the publication of reviews from the American [1] and British [2] Diabetic Associations concerning the dietary care of diabetic patients, there is a resurgence of interest in this field of therapy. Coupled with this, it is becoming increasingly evident that wherever possible antidiabetic therapy should return blood glucose levels to as near normal as possible if the development of diabetic vascular complications is to be ameliorated [3-5]. Therefore it seems important that the same critical analysis should be made of each form of anti-diabetic therapy, including diet.

In the majority of diabetic clinics, the practice is to commence therapy in Type 2 diabetic patients with diet alone for some months before considering further antidiabetic therapy. Diets used restrict either calories (energy) or carbohydrate (usually of the exchange type), and in both the carbohydrate content is prescribed to give $40 \%$ total calorie intake. This paper examines the results of this therapy and some of the factors which might influence results.

\section{Patients and Methods}

Over a period of 3 years, 214 patients were referred from general practitioners and other hospital clinics with untreated diabetes. Of these pa- tients, 32 ( 20 men and 12 women) failed, or were unable, to attend for follow-up due to age, immobility, intercurrent illness, mental infirmity or removal from Leeds.

All the 182 remaining patients ( 88 men, 94 women) completing 3 months' diet therapy suffered from untreated Type 2 diabetes. None was or had ever been known to be ketotic, nor had any received any prior dietary advice. No patient was receiving steroid therapy or the contraceptive pill, nor was any pregnant or suffering from any disease in which diabetes is a recognised complication, such as pancreatitis, thyrotoxicosis, etc.

A full clinical and family history and physical examination of each patient, including fundoscopy, was undertaken at the first visit. Body weight was expressed as a percentage of the ideal body weight for age, sex and height calculated from Kemsley's data [6].

Before treatment was commenced, an outpatient $50 \mathrm{~g}$, 2-h glucose tolerance test was performed with venous whole blood glucose levels measured by a glucose analyser (model 23AM, Clandon Scientific, Yellow Springs, Ohio, USA). The interassay relative standard deviation for blood glucose levels was $1.5 \%$. Plasma insulin levels were assayed during the glucose tolerance test by a double-antibody radioimmunoassay [7], all samples from one patient being measured in the same assay. The mean interassay relative standard deviation for plasma insulin assay was $8.3 \%(n=14)$. Total areas under the glucose and insulin curves were measured and expressed as mmol $\cdot 1^{-1} \cdot \mathrm{h}^{-1}$ or $\mathrm{mU} \cdot \mathrm{l}^{-1} \cdot \mathrm{h}^{-1}$. Fasting plasma triglyceride ('Technicon' $\mathrm{N}-78$ a method) and total cholesterol ('Technicon' N-77 method) levels were measured after an overnight fast. Interassay relative standard deviation for triglyceride was $6.8 \%$ and for cholesterol $4.5 \%$ (in both, $n=12$ ). During the glucose tolerance test all 182 patients studied had blood glucose levels $>5.5 \mathrm{mmol} / 1$ (fasting), $>10.0 \mathrm{mmol} / 1(1 \mathrm{~h})$ and $>6.6 \mathrm{mmol} / 1$ (2h). 
Table 1. Clinical details of 182 Type 2 diabetic patients before various dietary treatments

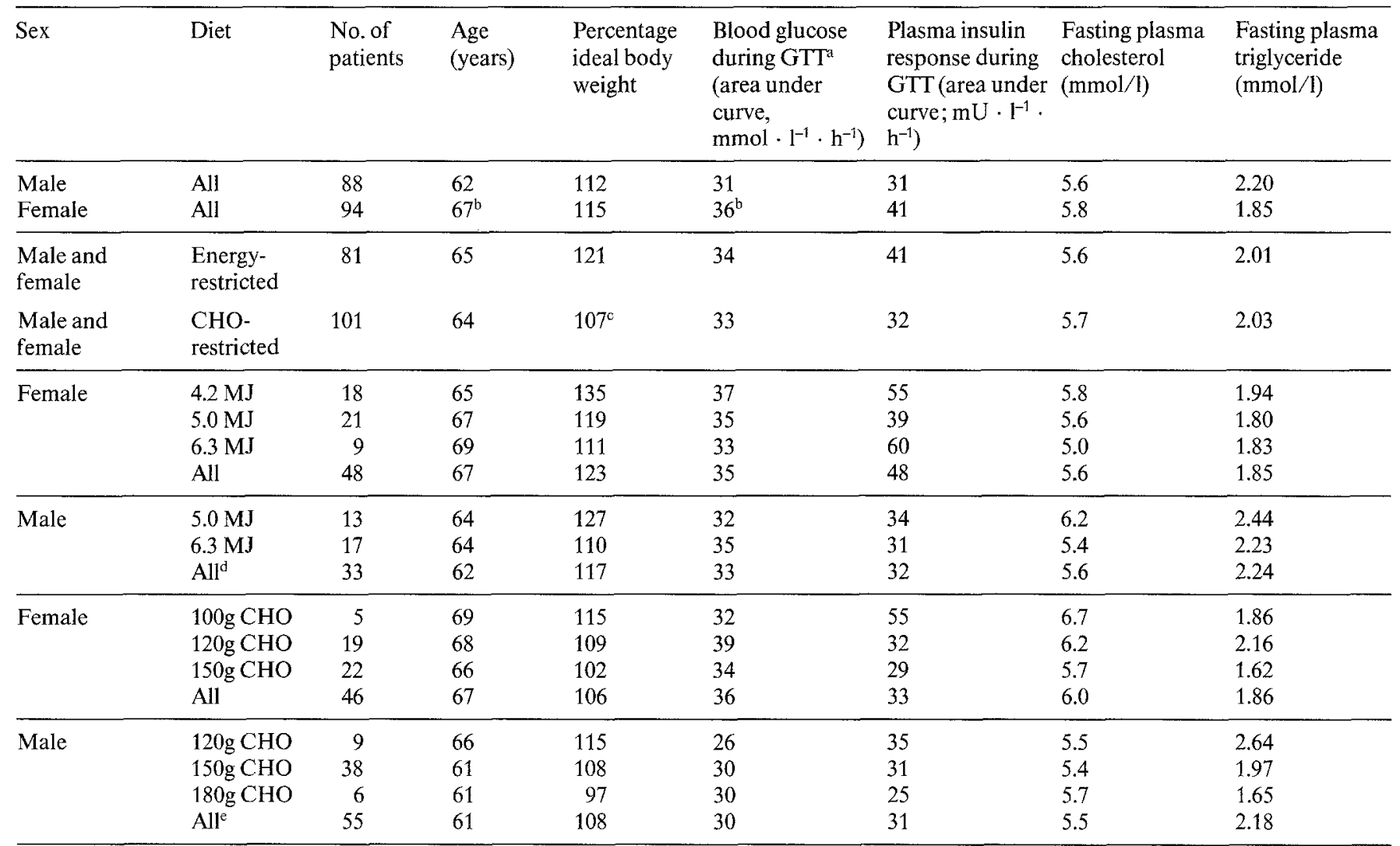

${ }^{a}$ GTT $=$ glucose tolerance test ${ }^{b} \mathrm{p}<0.05$ (difference between males and females) ${ }^{c} \mathrm{p}<0.05$ (difference between calorie-and CHO-restricted diets) ${ }^{d}$ Includes one patient on 4.2 MJ and two patients on 7.6 MJ diets e Includes two patients on 100-g CHO diet

\section{Dietary Regimens}

After the initial clinical assessment and glucose tolerance test, patients were allocated to a diet regimen chosen with regard to their physical activity, body weight and social position. Each diet was explained to the patient by a dietician and individual diet sheets given. Wherever possible meal patterns were preserved and wives of male patients were also interviewed by the dietician for explanation of diets. As a general policy mono- and di-saccharides were excluded from the diet but no firm advice was given concerning dietary fibre.

Two forms of dietary formulation were used: energy- (calorie) restrictive diets of $4.2 \mathrm{MJ} /$ day $(1000 \mathrm{kcal}), 5.0 \mathrm{MJ} /$ day $(1200 \mathrm{kcal}), 6.3 \mathrm{MJ} /$ day $(1500 \mathrm{kcal})$ or $7.6 \mathrm{MJ} / \mathrm{day}(1800 \mathrm{kcal})$; and carbohydrate- $(\mathrm{CHO}-)$ restricted diets of $100 \mathrm{~g} \mathrm{CHO}, 120 \mathrm{~g} \mathrm{CHO}, 150 \mathrm{~g} \mathrm{CHO}$ and $180 \mathrm{~g} \mathrm{CHO} /$ day in $10 \mathrm{~g}$ portions. The dietary prescribing policy in both diets was that $40 \%$ of calories in the diet were given as carbohydrate so that, for example, in a $6.3 \mathrm{MJ}$ diet the $\mathrm{CHO}$ content was $150 \mathrm{~g}$, and a $150 \mathrm{~g} \mathrm{CHO}$ diet contained 6.3 MJ. However the presentation of the two types of diet was quite different, with the emphasis on specific prescribing of food intake in the energy-restricted diets, compared with 'exchange list' prescribing in the carbohydrate-restricted diets. Of the $88 \mathrm{men}, 63 \%$ were prescribed $\mathrm{CHO}$-restricted diets and of the 94 women $51 \%$ were prescribed energy-restricted diets.

After instruction in urine testing with Clinitest reagent tablets, all patients were asked to test daily before breakfast and record the result.

The clinical details of the patients are shown in Table 1. The men were significantly younger than the women and had less glucose intolerance at diagnosis. However, in 50 pairs of men and women matched for age, there was no significant difference in glucose tolerance.

The majority of patients (54\%) presented with classical diabetic symptoms, including polyuria and polydipsia. Fourteen patients (all men) presented with no symptoms, glycosuria having been discovered at a routine medical examination (such as for insurance, or for heavy goods vehicle licence). Forty-seven patients were found to have glycosuria while attending other hospital clinics and referred directly to the diabetic clinic. Thirteen patients $(7 \%)$ were found to be suffering from diabetic retinopathy at diagnosis, and in four of these visual deterioration was the first symptom of the diabetes. However these variations in presentation made little difference to the measurements of each group except that the 13 patients with retinopathy were significantly lighter in weight and the group of 14 men without any symptoms had less glucose intolerance.

All patients were then seen monthly by the same doctor in an outpatient clinic in which they were weighed, urine charts were checked, symptoms were noted and any further examinations indicated were performed. Patients, with or without spouses, were also interviewed by the dietician to deal with any further dietary problems. After 3 months' therapy, the glucose tolerance test with plasma insulin and fasting plasma lipid levels was repeated while the patients were on their prescribed diet.

The results of therapy were analysed and compared using distribution-free statistical analysis [8], such as the Mann-Whitney ' $U$ ' test, Spearman rank correlation coefficient, Wilcoxon matched-pairs signed rank test and the $\chi^{2}$ test.

\section{Results}

In the 182 patients treated with these diet regimens for 3 months, there was on average a $25.4 \%$ improvement in glucose tolerance, a $58.1 \%$ increase in plasma insulin response during the glucose tolerance test, a $5.1 \%$ fall in 
Table 2. Effects of various dietary regimens for 3 months on weight loss, glucose tolerance, insulin response during glucose tolerance test (GTT) and fasting plasma lipid levels in 182 Type 2 diabetic patients

\begin{tabular}{|c|c|c|c|c|c|c|c|}
\hline Sex & Diet & $\begin{array}{l}\text { No. of } \\
\text { patients }\end{array}$ & \multicolumn{5}{|c|}{ Percentage change on treatment in: } \\
\hline Male & All & 88 & -4.9 & -30 & +55 & -3 & -20 \\
\hline Female & All & 94 & -5.3 & $-21^{b}$ & +61 & +1 & $-8^{b}$ \\
\hline Male and female & $\begin{array}{l}\mathrm{CHO} \\
\text { restricted }\end{array}$ & 101 & $-4.1^{c}$ & -24 & +54 & -2 & -18 \\
\hline Female & $\begin{array}{l}4.2 \mathrm{MJ} \\
5.0 \mathrm{MJ} \\
6.3 \mathrm{MJ} \\
\text { All }\end{array}$ & $\begin{array}{r}18 \\
21 \\
9 \\
48\end{array}$ & $\begin{array}{l}-6.9 \\
-4.9 \\
-6.4 \\
-6.2\end{array}$ & $\begin{array}{l}-26 \\
-22 \\
-15 \\
-22\end{array}$ & $\begin{array}{l}+148 \\
+\quad 36 \\
+\quad 5 \\
+\quad 75\end{array}$ & $\begin{array}{r}+7 \\
0 \\
-1 \\
+3\end{array}$ & $\begin{array}{l}-11 \\
-3 \\
+5 \\
-9\end{array}$ \\
\hline Female & $\begin{array}{l}100 \mathrm{~g} \mathrm{CHO} \\
120 \mathrm{~g} \mathrm{CHO} \\
150 \mathrm{~g} \mathrm{CHO} \\
\text { All }\end{array}$ & $\begin{array}{r}5 \\
19 \\
22 \\
46\end{array}$ & $\begin{array}{l}-7.2 \\
-4.2 \\
-3.5 \\
-4.5\end{array}$ & $\begin{array}{l}-18 \\
-19 \\
-24 \\
-21\end{array}$ & $\begin{array}{l}+41 \\
+41 \\
+53 \\
+46\end{array}$ & $\begin{array}{l}-5 \\
+1 \\
+2 \\
+1\end{array}$ & $\begin{array}{l}-14 \\
-13 \\
-14 \\
-14\end{array}$ \\
\hline Male & $\begin{array}{l}120 \mathrm{~g} \mathrm{CHO} \\
150 \mathrm{~g} \mathrm{CHO} \\
180 \mathrm{~g} \mathrm{CHO} \\
\mathrm{All}^{\mathrm{e}}\end{array}$ & $\begin{array}{r}9 \\
38 \\
6 \\
55\end{array}$ & $\begin{array}{l}-4.3 \\
-3.6 \\
-0.7 \\
-3.7\end{array}$ & $\begin{array}{l}-20 \\
-28 \\
-16 \\
-26\end{array}$ & $\begin{array}{l}+25 \\
+\quad 82 \\
-\quad 8 \\
+\quad 64\end{array}$ & $\begin{array}{l}+3 \\
-5 \\
+4 \\
-3\end{array}$ & $\begin{array}{l}-17 \\
-16 \\
+2 \\
-16\end{array}$ \\
\hline
\end{tabular}

a GTT: glucose tolerance test ${ }^{\mathrm{b}} p<0.05$ (difference between males and females) ${ }^{\mathrm{c}} p<0.05$ (difference between calorie and CHO) $\mathrm{d}$ includes one patient on 4.2 MJ and two patients on 7.6-MJ diets ${ }^{e}$ includes two patients on 100-g CHO diets

body weight, a $0.9 \%$ fall in fasting plasma cholesterol levels and a $13.6 \%$ fall in fasting plasma triglyceride levels.

Table 2 shows the effect of each diet regimen on glucose tolerance, insulin response during the glucose tolerance test, fasting plasma lipids and body weight. Overall there were no significant differences when energy-restricted and $\mathrm{CHO}$-restricted diets were compared except for a significantly greater weight loss on energy-restricted diets. However men as a whole had greater reductions in glucose tolerance and in fasting plasma triglyceride levels than did women. Both these effects were particularly marked in men given energy-restricted diets.

Table 3 shows the Spearman rank correlation coefficients between the percentage change in each of the following variables on the one hand, and the initial value of the same variable or change in other variables on the other hand: area under the glucose response curve, area under insulin response curve during the glucose tolerance test, fasting plasma lipid levels, and body weight. In men there were strong positive correlations between improvement in area under glucose response curve and both the reduction in body weight and fasting plasma triglyceride levels, particularly in men on CHO-restricted diets. In both men and women there was a strong negative correlation between the percentage changes in area under the glucose and insulin response curves during the glucose tolerance test, particularly in women on energy-restricted diets.

However, there were negative correlations between initial values and changes after therapy for the following variables: insulin response curve, weight (in men only), plasma cholesterol and triglyceride levels, and (weakly) area under blood glucose curve.

Of the 182 patients studied, 37 became well-controlled in that the area under the glucose response curve after treatment became normal $\left(<16 \mathrm{mmol}^{-1} \mathrm{l}^{-1} \cdot \mathrm{h}^{-1}\right)$. On the contrary, 29 patients were poorly-controlled as the area under their glucose response curves remained at least twice the upper limit of normal.

Those patients whose glucose tolerance became normal were mainly men, and were significantly heavier and had significantly less glucose intolerance before treatment than those patients who remained poorly controlled. On diet therapy the well-controlled patients lost more weight and showed a significantly greater fall in plasma triglyceride levels. Comparing the well- and poorly-controlled groups, there was no significant difference in the type of diet used (14 on energy-restricted and 23 on CHO-restricted diets in the well-controlled group and 10 and 19 respectively in the poorly-controlled group). 
Table 3. Spearman rank correlation coefficients between percentage changes in the following variables after 3 months' dietary therapy and pre-treatment levels of the same, or post-treatment levels of other variables in 88 male and 94 female Type 2 diabetic patients: body weight, glucose tolerance, insulin response during glucose tolerance test (GTT) and fasting plasma lipid levels

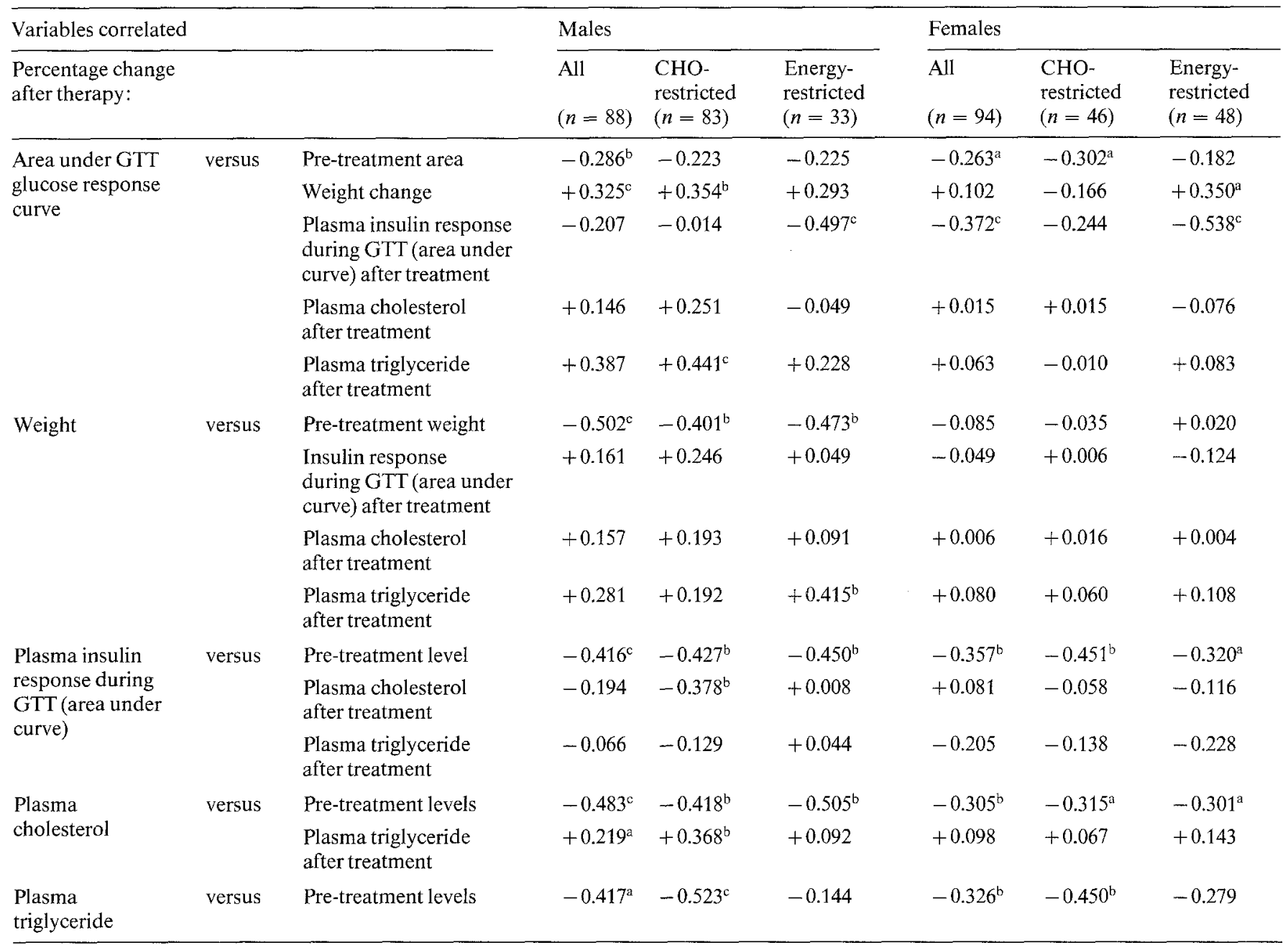

$\mathrm{a}<0.05{ }^{\mathrm{b}} \mathrm{p}<0.01 \quad \mathrm{c}<0.001$

To assess the effect of weight loss on diabetic control, two groups of patients were compared: one group of 25 patients (14 men) who lost more than $10 \%$ of their initial weight on diet, and a second group of 30 patients (21 men) who lost no weight $( \pm 1 \%$ of their initial weight). There were no significant differences between the groups in their pre-treatment details except that those who did not lose weight were lighter. However this was not due to this group having lost more weight before diagnosis. Those patients who lost weight on the diet showed a significantly greater improvement in glucose tolerance and a significantly greater fall in fasting plasma triglyceride levels, but no significant change in insulin response during the glucose tolerance test.

The effect of the patients' sex on their response to therapy was more difficult to assess because the women were significantly older and heavier. However, it was possible to match 29 pairs of men and women for age, obesity and type of diet prescribed. Improvement in glucose tolerance was then the same in men and women and the falls in plasma triglyceride levels were not signifi- cantly different. This suggests that the sex of the patient has no absolute effect upon the response to diet therapy.

Comparing the effects of energy-restricted and CHO-restricted diets, patients on the former diets lost more weight despite the theoretical energy parity of the diets, but there were no significant differences in the percentage changes in the other measurements. However patients prescribed energy-restricted diets were heavier at diagnosis. If patients were matched for initial body weight and for $\mathrm{CHO}$ and energy content of their selected diets then there were no significant differences.

From the 182 patients in this study, it was possible to identify two groups with different responses in plasma insulin levels during a glucose tolerance test. A first group of 30 patients more than doubled their insulin response during a glucose tolerance test after dietary therapy, and a second group of 57 patients responded with a fall in plasma insulin levels (Table 4). These two groups had equal numbers of men and women and were comparable for age, weight and family history of diabetes. However, those patients in whom therapy pro- 
Table 4. The pre-treatment clinical details and the response to 3 months' dietary therapy in two groups of Type 2 diabetic patients: group 1 showing a fall in insulin response during glucose tolerance test (GTT) on therapy, and group 2 showing an increase in insulin response of $>100 \%$

\begin{tabular}{|c|c|c|c|c|c|c|c|c|c|}
\hline & \multicolumn{5}{|c|}{ Pre-treatment details } & \multicolumn{4}{|c|}{ Percentage change after treatment in: } \\
\hline Group I: & \multicolumn{9}{|c|}{ Patients with a fall in insulin secretion on treatment ( $n=57,28$ male: 29 female) } \\
\hline \multirow[t]{2}{*}{ Group 2: } & \multicolumn{9}{|c|}{ Patients with increase in insulin secretion $>100 \%(n=30,15$ male, 15 female $)$} \\
\hline & 65 & 113 & $53 \%$ & $37^{\mathrm{a}}$ & $19^{\mathrm{b}}$ & -5.0 & $-37^{c}$ & -5 & -18 \\
\hline
\end{tabular}

duced a fall in plasma insulin levels had significantly less glucose intolerance and greater plasma insulin response during the pre-treatment glucose tolerance test. In this group, diet therapy produced a significantly smaller percentage fall in glucose tolerance but no difference in weight loss or plasma lipid changes compared with the group in whom diet therapy increased plasma insulin levels. Each group had similar types of diets prescribed and similar numbers of patients who achieved normal glucose tolerance.

After 3 months' diet therapy, $47 \%$ of patients on energy-restricted diets and $46 \%$ of those on CHO-restricted diets had detected no glycosuria on home urine testing; $16 \%$ and $21 \%$ respectively had occasional glycosuria and $37 \%$ and $34 \%$ respectively had regular glycosuria. As might be expected, those patients with regular glycosuria had significantly greater glucose intolerance after therapy. After dietary therapy, $61 \%$ patients had no diabetic symptoms, $23 \%$ had occasional symptoms and $17 \%$ still complained regularly of symptoms referrable to their diabetes. Again those patients with symptoms had significantly greater glucose intolerance after treatment compared with those patients without symptoms.

\section{Discussion}

In a lecture given in 1972 reviewing the dietetic treatment of diabetes Pyke [9] ended by reminding his audience of the degree of ignorance that existed about the effects of such therapy. West [10] described in strong terms in his review of diet therapy the pitfalls and difficulties experienced by physician and patient alike in formulating and complying with dietary advice. The same point had been made earlier in Leeds by Tunbridge and Wetherill[11].

This present study made no particular point of stressing or measuring compliance except by the usual means of clinical practice. Indeed it was felt that the follow-up of these patients should not differ in any way from normal clinical practice, so as to avoid the criticism that the results were obtained from a small, selected popula- tion of compliant patients undergoing intense diet control bearing little relationship to usual clinical practice. The results of diet therapy alone in these patients might be considered as being disappointing. However, two provisos should be borne in mind. First, patients with mild carbohydrate intolerance, such as those with normal fasting but raised 2-h blood glucose levels, were not included. Many of those patients would have been expected to respond to diet alone. Secondly a return to normal glucose tolerance may not be required to prevent the progression of diabetic vascular complications [12]. However, there seems no doubt that the results of diet therapy could be improved by a more intensive education programme as described by Hadden et al. [13], particularly with the use of domiciliary dietetic services.

This study followed the practice of prescribed diabetic diets to restrict either overall energy (calorie) intake or carbohydrate intake, and in both types of diet the carbohydrate content was maintained at $40 \%$ total calories. Thus the diets were theoretically similar. From the practical point of view energy-restricted diets are more difficult to manage in respect of patient compliance and involvement of dieticians' time and skill. However, this study has shown that, if improvement in glucose tolerance alone is required, then either an energy- or a CHOrestricted diet would be equally effective, but if weight loss is also required then an energy-restricted diet is more likely to be effective. This difference in weight-loss no doubt reflects the inadvertent intake of higher amounts of total calories on the $\mathrm{CHO}$-restricted diets arising from the increased use of low-CHO foods, such as cheese. The lack of response in serum cholesterol levels presumably reflects the higher fat intake in both diets, and contrasts with the marked changes in fasting plasma triglyceride levels due to the restriction of carbohydrate to $40 \%$ of total calories.

This study measures the overall response to these diets as prescribed, in Type 2 diabetic patients. It is hoped that in the near future it will be possible to compare these results with those gained from similar patients given diets containing a higher percentage of carbohydrate and/or less-available sources of carbohydrate. 
Acknowledgements. I am grateful to Sister Bilborough and her staff in Medical Outpatients for all their help, and to Mrs. B. M. Edwards for her technical assistance with this study.

\section{References}

1. Committee of the American Diabetes Association on Food and Nutrition: Special Report (1979) Diabetes Care 2: 520-523

2. Nutrition Sub-committee of the Medical Advisory Committee, British Diabetic Association (1980) Dietetic recommendations for diabetics for the 1980's

3. Anon (1979) Good diabetic control lessens chronic complications. Drug Therap Bull 17:93-94

4. Miki E, Fukuda M, Kuzuya T, Kosada K, Najao K (1969) Relation of the course of retinopathy to control of diabetes, age and therapeutic agents in diabetic Japanese patients. Diabetes 18:773-780

5. Ward JD, Barnes CG, Fischer DJ, Jessop JD, Baker RWR (1971) Improvement in nerve condition following treatment in newly diagnosed diabetics. Lancet 1:428-430

6. Kemsley WFF (1951) Body weights and different ages and heights. Ann Eugenics 16:316-334

7. Morgan CR, Lazarow A (1963) Immunoassay of insulin. Diabetes 12: $115-126$
8. Siegel S (1956) Non-parametric statistics for the behavioural sciences. McGraw-Hill Kogaksha Ltd, Tokyo

9. Pyke DA (1972) The dietetic treatment of diabetes: current aspects. Acta Diabet Lat 9: suppl 1, 511-523

10. West KM (1978) Diet therapy of diabetes: an analysis of failure. Ann Int Med 79:425-434

11. Tunbridge RE, Wetherill JH (1970) Reliability and costs of diabetic diets. Br Med J 2: 78-82

12. Jarrett RJ, Keen H (1976) Hyperglycaemia and diabetes mellitus. Lancet 2: 1009-1012

13. Hadden DR, Mongomery DAD, Skelly RJ, Trimble ER, Weaver JA, Wilson EA, Buchanan KD (1975) Maturity-onset diabetes mellitus: response to intensive dietary management. Br Med J 3: $376-378$

Received: 2 November 1981

and in revised form: 20 May 1982

Dr. John K. Wales,

University Department of Medicine,

Martin Wing

General Infirmary,

Leeds LS1 3EX, UK 